\title{
Ecogenomic survey of plant viruses infecting Tobacco by Next generation sequencing
}

\author{
Ibukun A. Akinyemi ${ }^{1 \dagger}$, Fang Wang ${ }^{2 \dagger}$, Benguo Zhou ${ }^{2}$, Shuishui Qi and Qingfa Wu ${ }^{1 *}$
}

\begin{abstract}
Background: The invasion of plant by viruses cause major damage to plants and reduces crop yield and integrity. Devastating plant virus infection has been experienced at different times all over the world, which are attributed to different events of mutation, re-assortment and recombination occurring in the viruses. Strategies for proper virus management has been mostly limited to eradicating the vectors that spreads the plant viruses. However, development of prompt and effective diagnostic methods are required to monitor emerging and re-emerging diseases that may be symptomatic or asymptomatic in the plant as well as the genetic variation and evolution in the plant viruses. A survey of plant viruses infecting field-grown Tobacco crop was conducted in Anhui Province of China by the deep sequencing of sRNAs.

Methods: Survey of plant viruses infecting Tobacco was carried based on 104 samples collected across the province. Nine different sRNA libraries was prepared and custom-made bioinformatics pipeline coupled with molecular techniques was developed to sequence, assemble and analyze the siRNAs for plant virus discovery. We also carried out phylogenetic and recombination analysis of the identified viruses.

Results: Twenty two isolates from eight different virus species including Cucumber mosaic virus, Potato virus $Y$, Tobacco mosaic virus, Tobacco vein banding Mosaic virus, Pepper mottle virus, Brassica yellow virus, Chilli venial mottle virus, Broad bean wilt virus 2 were identified in tobacco across the survey area. The near-complete genome sequence of the 22 new isolates were determined and analyzed. The isolates were grouped together with known strains in the phylogenetic tree. Molecular variation in the isolates indicated the conserved coding regions have majorly a nucleotide sequence identity of $80-94 \%$ with previously identified isolates. Various events of recombination were discovered among some of the isolates indicating that two or more viruses or different isolates of one virus infect the same host cell.
\end{abstract}

Conclusion: This study describes the discovery of a consortium of plant viruses infecting Tobacco that are broadly distributed in Anhui province of China. It also demonstrates the effectiveness of NGS in identifying plant viruses without a prior knowledge of the virus and the genetic diversity that enhanced mixed infection.

Keywords: sRNA, Bioinformatics, Assembly, Next generation sequencing, Plant virus

\footnotetext{
* Correspondence: wuqf@ustc.edu.cn

${ }^{\dagger}$ Equal contributors

'School of Life Sciences, University of Science and Technology of China,

Hefei, Anhui 230027, China

Full list of author information is available at the end of the article
} 


\section{Background}

Viruses are ubiquitous and affects plant growth and yield. Over the years, crop losses due to viral infection have been very devastating and are of great concern especially in developed and developing countries [1]. The unrestricted distribution of plant diseases and emerging infectious disease poses serious threat to food sustenance. A high percentage of this loss is caused by viruses because of their abundant presence in most environments among the biological entities [2]. Early identification of these plant pathogens remains the focal point in the field of virology, aimed at preventing the spread of the viruses as well as developing ways of combating and reducing their effects on agricultural yield.

RNA viruses exists within host as a consortium of unidentical but similar sequences due to their inability to propagate without a living host, high mutation and recombination rates, referred to as viral quasispecies [3]. RNA silencing is one of the defense mechanisms of plant against viruses, in which the double stranded RNA (dsRNA) serves as a substrates for Dicer-like ribonuclease (DCL) to produce small-interfering RNAs (siRNAs) of between 21 to $25 \mathrm{nt}$ [4]. Viral infection of plants involves the production of viral small RNAs (vsRNAs) and the plant host interacts with invading viruses by developing various cellular mechanisms. Viruses are both inducers and targets of RNAi [5]. Double stranded RNA intermediates are produced by viral genomes during replication which serves as substrates for Dicer-like ribonucleases and cleaves into small virus derived siRNAs (viRNAs), and binds with the Argonaut protein (Ago) to form the RISC complex [6].

The advent of new sophisticated technologies for parallel sequencing had increased our understanding of viral genome variability and evolution within the host and virus defense mechanism in plants. It is widely accepted that studies of viral abundance and diversity will lead and have led to novel insights into the functioning of the microbial biosphere. The relative abundance of a virus (or viral nucleic acid) in a sample, compared to that of other organisms such as bacteria or host cells (or their genomes), is a critical factor for the discovery of viruses when using metagenomics. Unlike traditional virus detection methods e.g., enzyme-linked immunosorbent assay (ELISA), polymerase chain reaction (PCR), or microarray which depends on prior knowledge of antibody or sequence of the potential virus [7] as well as determining the existence of novel viral agents $[8,9]$, the use of next generation sequencing technology (NGS) provides a powerful method for determining the causative pathogen without the prior knowledge of the disease pathogen. The genome of plant viruses can be rapidly determined even when occurring at extremely low titers in the infected host. The detection of both DNA and RNA viruses [10] has been made possible by the reconstruction of partial or complete viral genomes [11] and sequencing of the accumulated 21-24 nt virus-derived siRNAs generated by Dicer enzymes upon recognition of viral dsRNA. With the development of NGS technology and its relatively low cost, NGS has widen understanding and its potentials of diagnostics of viral pathogens without a priori knowledge of the invading pathogen, which provide accurate and timely detection of these viral pathogens in plant for effective disease management and control. Consequently, it is necessary to conduct an accurate and timely detection of these viral pathogens in plant for effective disease management and control.

Tobacco (Nicotiana tabacum) is an important economic crop worldwide, with half of the world's tobacco farmers in China and the world's largest producer [12], ahead of countries such as India, Zimbabwe, Indonesia, Turkey, Bangladesh, Egypt, Philippine and Thailand. The production and yield of tobacco have been seriously affected by the invasion of emerging and recurrent plant viruses with symptoms such as venial necrosis, mosaic, mottling, yellowing, ring spots, stunting, shoestring and deformation [13-16]. Anhui province is in the center of China and surrounded by six other provinces. The typical geographical feature of this area is enriched with geochemical elements suitable for the flourishing of Tobacco plant [17]. The tobacco plantation is usually surrounded or mixed with other crops, as mixed system of farming is commonly adopted in these areas. These however, had enhanced the transmission of plant virus from one plant to the other and consequently made Anhui province an idealistic open ecosystem to investigate the viruses infecting the crop in the province. In order to identify the etiological agents of the different disease symptoms observed in different Tobacco plantation across Anhui province, we used next generation sequencing of small RNAs to identify viruses from symptomatic Tobacco plants in farm fields. We also present the results of genome comparison between the resulting 22 isolates and genomes retrieved from GenBank. This study provides census of viral population and distribution in different ecosystem or cropping system through the characterization, discovery and molecular interaction of plant viruses. We also described the recombination events that occurred in the isolates and a bioinformatics pipeline that explores the siRNA generated in response to viral invasion and other molecular biology methods employed to discover a consortium of viruses infecting tobacco.

\section{Results}

\section{Sequence data}

One hundred and four symptomatic leaf samples of tobacco were collected from farm fields and pooled into 9 combinations based on the location of sample collected. 
Nine individual sRNA libraries were constructed and sequenced on an Illumina HiSeq-2000 platform which generated between 38 and 49 million raw reads. Adapters and low quality reads were trimmed to obtain between 32 and 45 million clean reads with lengths of 18-30 nt (Tables 1). The most abundant class of viral derived small RNAs were 21-24 nt in length, with a peak of $24 \mathrm{nt}$ for all samples (Additional file 1: Figure S1). Similar length distribution of sRNA were observed in all the libraries (Additional file 1: Figure S2a,b,c).

\section{Virus identification}

The homology-dependent approach for plant virus discovery was employed for the analysis, using a custom-made pipeline (Fig. 1). The clean reads of each library were assembled into contigs with Velvet [18] and Oases [19]. The number of contigs produced were 6346-8981 (Table 1) based on the de-bruijn graph algorithm ( $k$-mer 17$)$, with the longest single contig length of 2,255 nt. GC content of the assembled contigs ranges between 43 and $45 \%$. These contigs were searched against the non-redundant nucleotide sequence entries of the National Center for Biotechnology Information (NCBI) database by BLASTn with e-value of $10^{-3}$. Stringent parameters of $\geq 90 \%$ identity and coverage identified 554 contigs hit distantly related to sequences in the database at nucleotide and protein level (Table 2). Related reference genome sequences were used to determine the relative positions and orientation of these final contigs. Subsequently, RT-PCR was performed to obtain the near-complete genome sequence of all the isolates using primers designed based on the final contigs. The genome sequence of identified viruses from all the libraries have high nucleotide sequence identity with respective matching genome from the Genbank database (Table 3). BLASTn search of the full-length nucleotide sequence against the database indicated the assembled genomes from the libraries have significant similarities to viruses belonging to Cucumovirus, Tobamovirus, Polerovirus and Potyvirus of the Family
Bromoviridae, Virgaviridae, Luteoviridae and Potyviridae respectively (Table 3 ). Specifically, 8 different virus species were identified which includes Cucumber mosaic virus (CMV), Potato virus $Y$ (PVY), Tobacco mosaic virus (TMV), Tobacco vein banding Mosaic virus (TVBMV), Pepper mottle virus (PeMV), Brassica yellow virus, Chilli venial mottle virus (CVMV), Broad bean wilt virus 2 (BBWV) infecting tobacco across the survey area. In order to gain insight into the host RNA silencing mechanism induced by the invading viruses, analysis of the sequences characterized by Bowtie [20] allowing no mismatch showed an equal distribution of siRNAs along the genome of the new virus while no excess of $(+)$ over $(-)$ siRNAs (Additional file 1: Figure S3) but revealed a peak of 22 and 24 nts in the genome sequence. The genome sequence of the 22 isolates from this study has been deposited at the GenBank database under the accession number KX650847-KX650868.

\section{Genome properties}

The single protein gene 1a responsible for viral replication was predicted in the CMV isolates Xch-1, Xch-2, Xch-Wh, Bzh-2, Bzh-3, Bzh-4, Bzh-5 (accession number KX650847, KX650848, KX650849, KX650850, KX650851, KX650852, KX650853 respectively) by the ORF Finder. The gene is a major virulence factor of the cucumber mosaic virus (CMV) and its essential for replication of the viral genome [21]. The ORF 1a encodes 993 amino acids (aa) protein, known to be involved in replication. The conserved domain of the putative ORF 1a-encoded protein of the CMV isolates are also found in association with two enzymatic motifs: viral methyl transferase and viral helicase consistent with other cucumoviruses [22]. Also, a large ORF 1a of the CMV isolates encoded the virus replicase protein of between 533 and 933 amino acids (aa) with an estimated molecular mass of $\sim 45-112 \mathrm{kDa}$ consistent with other cucumoviruses. The 7 isolates of the CMV have high nucleotide similarity with the reference genome of isolate Fny (D00356). CMV-Xch-1 (KX650847)

Table 1 Description of Next generation sequencing data from one hundred and four samples

\begin{tabular}{lllllll}
\hline Library Source (City) & Library Name & No of Samples & Raw Reads & Clean reads & Unique Seq & No of Contigs Assembled by Velvet $(k=17)$ \\
\hline Xuancheng & Xch 1 & 14 & $44,729,605$ & $38,973,127$ & $7,051,733$ & 7952 \\
& Xch 2 & 11 & $49,938,373$ & $45,290,836$ & $7,131,823$ & 7038 \\
Xuancheng/Wuhu & Xch-Wh & 5 & $38,747,172$ & $34,282,436$ & $5,985,886$ & 6346 \\
Dongzhi/Qingyang & Dzh-Qyg & 11 & $43,827,189$ & $38,882,338$ & $6,522,149$ & 6776 \\
Bozhou & Bzh 1 & 17 & $45,974,903$ & $39,218,493$ & $7,582,592$ & 8133 \\
& Bzh 2 & 11 & $41,574,669$ & $36,303,201$ & $7,641,767$ & 8981 \\
& Bzh 3 & 9 & $43,324,495$ & $37,668,456$ & $6,579,416$ & 7388 \\
& Bzh 4 & 8 & $41,720,630$ & $37,414,819$ & $6,770,175$ & 8011 \\
& Bzh 5 & 18 & $38,277,531$ & $32,358,941$ & $6,088,147$ & 6698 \\
\hline
\end{tabular}




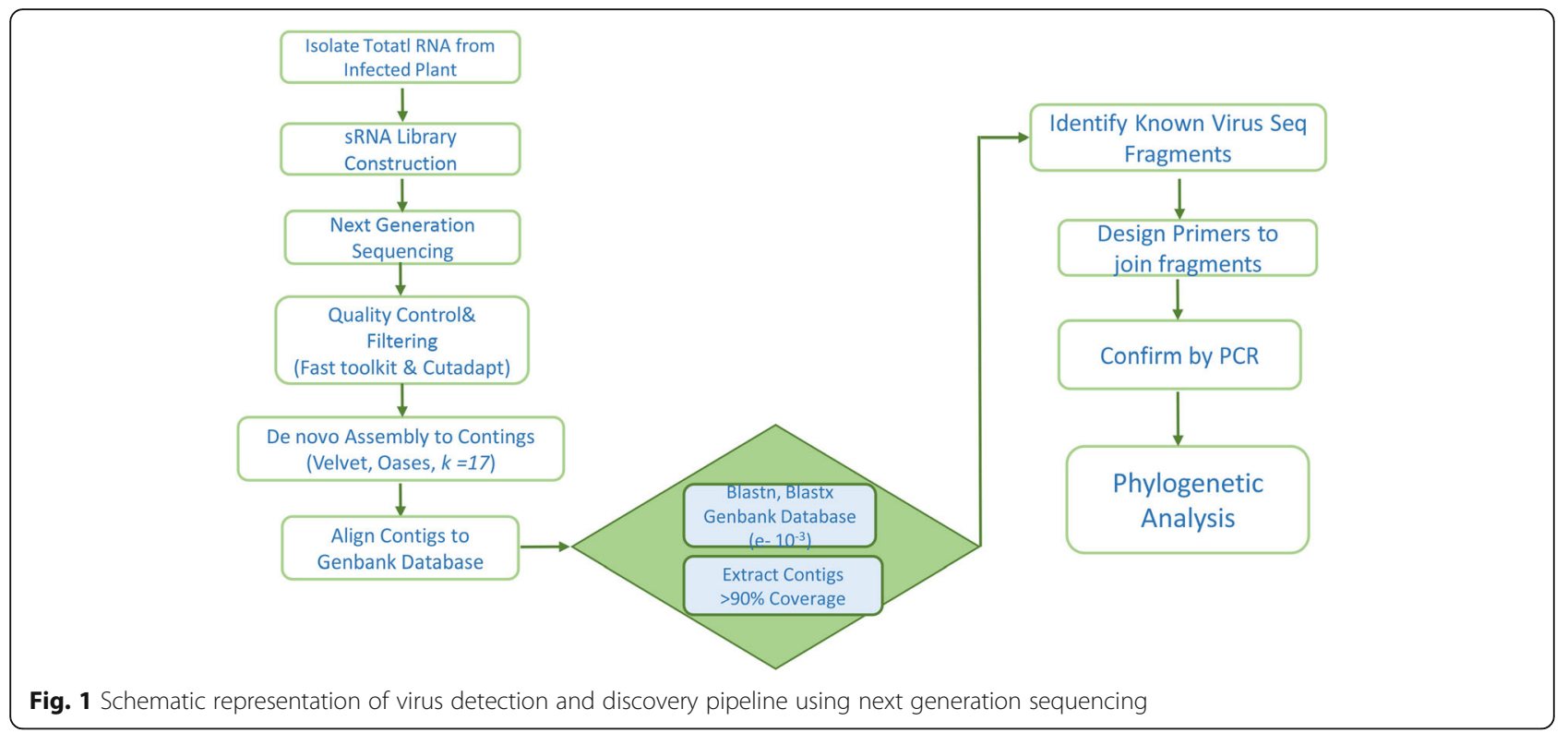

Table 2 Analysis of the assembled contigs against sequences in the NCBI database, using a highly homology sequence search

\begin{tabular}{|c|c|c|c|c|c|c|c|}
\hline Library Source & Library Name & Viruses & $\begin{array}{l}\text { No of contigs identical } \\
\text { to Blastn }\end{array}$ & Size (nt) & $G+C \%$ & Mapped Organism & $\begin{array}{l}\text { Relative } \\
\text { (ref Seq) }\end{array}$ \\
\hline \multirow[t]{5}{*}{ Xuancheng } & \multirow[t]{3}{*}{ Xch 1} & CMV-Xch-1 & 43 & 2874 & 45.95 & Cucumber Mosaic Virus & 3357 \\
\hline & & PVY-Xch-1 & 20 & 9292 & 42.53 & Potato Virus Y & 9704 \\
\hline & & PeMV-Xch & 14 & 9632 & 43.42 & Pepper Mottle Virus & 9640 \\
\hline & \multirow[t]{2}{*}{ Xch 2} & CMV-Xch-2 & 24 & 2922 & 44.92 & Cucumber Mosaic Virus & 3357 \\
\hline & & TMV-Xch-2 & 23 & 4679 & 42.87 & Tobacco Mosaic Virus & 6395 \\
\hline \multirow[t]{4}{*}{ Xuancheng/Wuhu } & \multirow[t]{4}{*}{ Xch-Wh } & CMV-Xch-Wh & 5 & 3102 & 45.34 & Cucumber Mosaic Virus & 3357 \\
\hline & & PVY-Xch-Wh & 14 & 9302 & 42.02 & Potato Virus Y & 9704 \\
\hline & & BrYV-Xch-Wh & 12 & 3494 & 42.86 & Brassica Yellow Virus & 5666 \\
\hline & & TMV-Xch-Wh & 31 & 5808 & 43.10 & Tobacco Mosaic Virus & 6395 \\
\hline \multirow[t]{2}{*}{ Dongzhi/Qingyang } & \multirow[t]{2}{*}{ Dzh-Qyg } & TMV-Dzh-Qyg & 33 & 5571 & 43.10 & Tobacco Mosaic Virus & 6395 \\
\hline & & CVMV-Dzh-Qyg & 20 & 9499 & 41.08 & Chilli venial mottle virus & 9711 \\
\hline \multirow[t]{12}{*}{ Bozhou } & Bzh-1 & PVY-Bzh-1 & 21 & 8826 & 42.18 & Potato Virus Y & 9704 \\
\hline & Bzh-2 & CMV-Bzh-2 & 29 & 2786 & 38.42 & Cucumber Mosaic Virus & 3357 \\
\hline & \multirow[t]{3}{*}{ Bzh-3 } & PVY-Bzh-3 & 34 & 9560 & 42.46 & Potato Virus Y & 9704 \\
\hline & & CMV-Bzh-3 & 18 & 2754 & 45.51 & Cucumber Mosaic Virus & 3357 \\
\hline & & TvBMV-Bzh-3 & 41 & 9148 & 42.15 & Tobacco Vein Banding Mosaic virus & 9570 \\
\hline & \multirow[t]{3}{*}{ Bzh-4 } & PVY-Bzh-4 & 37 & 9412 & 42.11 & Potato Virus Y & 9704 \\
\hline & & CMV-Bzh-4 & 16 & 2836 & 45.89 & Cucumber Mosaic Virus & 3357 \\
\hline & & TvBMV-Bzh-4 & 31 & 9449 & $42 . .12$ & Tobacco Vein Banding Mosaic virus & 9570 \\
\hline & \multirow[t]{4}{*}{ Bzh-5 } & CMV-Bzh-5 & 24 & 2967 & 46.22 & Cucumber Mosaic Virus & 3357 \\
\hline & & BBWV2-Bzh-5 & 29 & 3106 & 43.59 & Broad Bean Wilt Virus 2 & 3607 \\
\hline & & TvBMV-Bzh-5 & 35 & 9312 & 41.97 & Tobacco Vein Banding Mosaic virus & 9570 \\
\hline & & Total $=$ & 554 & & & & \\
\hline
\end{tabular}

Note: Reference used were retrieved from the NCBI database Cucumber mosaic virus (NC_002034.1), Potato virus Y(NC_001616.1), Tobacco mosaic virus(NC_001367.1), Tobacco vein banding Mosaic virus(NC_009994.1), Pepper mottle virus(NC_001517.1), Brassica yellow virus(NC_016038.1), Chilli venial mottle virus(NC_005778.1), Broad bean wilt virus 2(NC_003004.1) 
Table 3 Percentage nucleotide identity of identified viruses in each library to reference genome from NCBI database

\begin{tabular}{|c|c|c|c|c|c|c|c|c|c|}
\hline Virus Identified & Xch 1 & Xch 2 & Xch-Wh & Dzh-Qyg & Bzh 1 & Bzh 2 & Bzh 3 & Bzh 4 & Bzh 5 \\
\hline Cucumber mosaic virus & 77.34 & 77.58 & 84.63 & - & - & 62.64 & 75.07 & 77.95 & 80.25 \\
\hline Potato virus $Y$ & 89.15 & - & 84.78 & - & 81.67 & - & 83.88 & 88.00 & - \\
\hline Tobacco Mosaic virus & - & 72.06 & 89.31 & 86.11 & - & - & - & - & - \\
\hline Tobacco Vein Banding Mosaic virus & - & - & - & - & - & - & 87.06 & 89.40 & 88.61 \\
\hline Pepper Mottle Virus & 94.18 & - & - & - & - & - & - & - & - \\
\hline Chilli venial mottle virus & - & - & - & 78.70 & - & - & - & - & - \\
\hline Brassica Yellow Virus & - & - & 52.97 & - & - & - & - & - & - \\
\hline Broad Bean Wilt Virus 2 & - & - & - & - & - & - & - & - & 72.48 \\
\hline
\end{tabular}

-: Not available

shares $79 \%$ nucleotide sequence identity with CMV-Ix isolate from the Philippines (U202201), while CMV-Bzh-4 (KX650852) had $80 \%$ identity with the CMV-SD isolate (AF071551) from China.

The PVY isolates contain a single large ORF predicted by ORF Finder, consistent with other members of the potyvirus. The ORF of PVY-Xch-1, PVY-Xch-Wh, PVYBzh-1 isolates (accession number KX650858, KX650859, KX650860) are 166-7,362, 2,568-9,095 and 2,723-8,824 nt and encodes a polyprotein of 2,398, 2,175 and 2,033 aa with an estimated molecular mass of $\sim 272,248$ and $231 \mathrm{kDa}$ respectively. Comparison of PVY isolates polyprotein aa sequence with those of other PVY strains showed the presence of nine predicted cleavage sites, generating ten mature proteins (P1, HC-Pro, P3, $6 \mathrm{~K} 1, \mathrm{CI}$, $6 \mathrm{~K} 2, \mathrm{Vpg}$, NIa-Pro, NIb, and CP) after processing by virus-encoded proteases [23]. PVY-Bzh-1 (KX650860) has $90 \%$ nucleotide identity with isolate PVY-Guiding-3 (HM590405), representing an a close similarity between the sequences. The ORF predicted in pepper mottle virus (PeMV-Xch) isolate (KX650857) reveals a single long polyprotein between 2,423 and 7,345 nt, also typical of potyviruses of the family Potyviridae [24]. PeMv-Xch encodes 1,640 aa, estimated to have a molecular weight of $186 \mathrm{kDa}$. This share $94 \%$ nucleotide identity with the isolate (NC_001517.1) from USA.

Three isolates were identified to be Tobacco mosaic virus from samples collected in Xuancheng, Dongzhi, Qingyang and Bozhou. TMV has two replication-related genomic protein segment of 126 and $183 \mathrm{kDa}, 30-\mathrm{kDa}$ movement protein (MP) and a $17.5-\mathrm{kDa}$ coat protein (CP). TMV-Xch-2 (KX650854) isolate encodes replicase protein between the 3 and 4,127 nt, and a movement protein encoded between 4,111 and 4,677 nt. Also the replicase and movement protein of TMV-Dzh-Qyg (KX650856) isolate are encoded between 144 and 4,585 nt and 4,569-5,375 nt respectively. TMV-Xch-Wh (KX650855) however has additional coat protein encoded between 5,410-5,808 nt expressed from a second subgenomic RNA [25].

\section{Phylogenetic analysis of Isolates}

The identified near-complete nucleotide sequences in this study, other previously identified isolates as well as major strains of species with large group members were retrieved from the GenBank database and used to determine the phylogenetic relationships between the isolates and members of the same genus. This is important to understand plant virus evolution and events such as recombination and reassortment that are major evolutionary force in virus population [26]. Neighbor-joining phylogenetic analysis based on full-length viral genomes was performed with a bootstrap of 1000 replications. The genomic sequence of the $7 \mathrm{CMV}$ isolates (Table 2) shared a single 1a gene of between 80 and $95 \%$ identity with other isolates. The isolates were grouped into clusters according to the sequences of the closest relatives in the genus (Fig. 2). All the selected sequences of the cucumoviruses and identified isolates were well separated into three subgroups (IA, IB and II). The new cucumber mosaic virus isolates from Anhui province, China formed clusters with some proven standard isolates that were taken as reference isolates for each subgroup such as isolates Fny, MF and Y for subgroup IA; isolates CTL, NT9, SD and TFN for subgroup IB; isolates LY, Q, S and Trk7 for subgroup II, which are consistent with previous studies [22, 27]. The divergence of subgroups I and II is clearly seen in the 1a phylogeny estimation (Fig. 2); however, further divergence of subgroup I into IA and IB is not obvious but indicates an evolutionary history that is quite different from what is seen in the other RNAs. The 87.24\% sequence identity of isolate CMV-Xch-1 (KX650847) and CMV-Xch-2 (KX650848) formed the same cluster in the subgroup $1 \mathrm{~b}$ while the CMV-Bzh-3 (KX650851) and CMV-Bzh-5 (KX650853) isolates from different county in Bozhou form separate cluster with $90 \%$ nucleotide identity in the same subgroup. However, the CMV-Bzh-4 (KX650852) had 90.12\% identity with the CMV-SD (AF071551.1) isolate while CMVBzh-2 is the only isolate in the subgroup II, indicating that most isolates from the China survey are clustered in subgroup 1 (Fig. 2). 


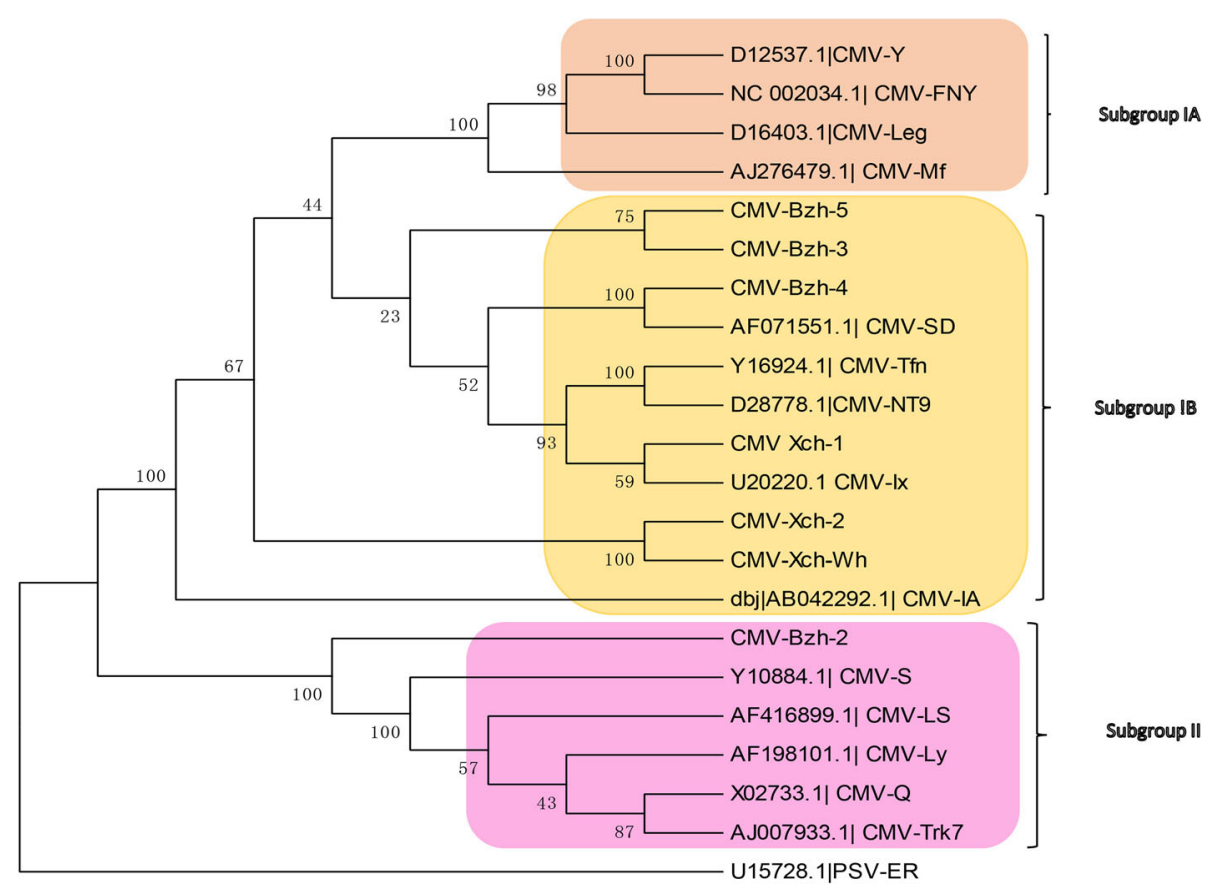

Fig. 2 Phylogenetic analyses of Cucumber mosaic virus Isolates and selected strains, with aligned nucleotide sequences, generated using the neighbor-joining method and MEGA6 software. The percentage of replicate trees in which the associated taxa clustered together in the bootstrap test (1000 replicates) is shown next to the branches. Peanut stunt virus ER RNA 1 was used as an out group for the tree construction

As for the Potyviruses, 5 isolates formed the same clade as the potato virus Y (NC_001616.1). The strains of Potato virus $\mathrm{Y}$ are divided into three groups $\left(\mathrm{PVY}^{\mathrm{C}}, \mathrm{PVY}^{\mathrm{O}}, \mathrm{PVY}^{\mathrm{N}}\right)$ $[28,29]$ and recombinant variants $\left(\mathrm{PVY}^{\mathrm{NTN}}\right)$ according to biological properties and genome sequence [30, 31]. PVY isolates Bzh-1 (KX650860), Bzh- 3 (KX650861), Bzh-4 (KX650862) and Xch-Wh (KX650859) clusters within the NTN group while the PVY-Xch-1 (KX650858) isolate falls within the PVY O strain group (Fig. 3). PVY-Xch-1 (KX650858) is closest to the PVY-Oz isolate (EF026074) from the USA with 90 \% nucleotide identity. The PVY isolates have shown great ability to adapt to different changing environmental conditions throughout the world probably because of the genetically diverse populations of the RNA viruses [32]. Most PVY isolates are clustered in the $\mathrm{PVY}^{\mathrm{NTN}}$ subgroup which has been regarded as the group of recombinant variant of $\mathrm{PVY}^{\mathrm{N}}$. This can be explained by a $94 \%$ nucleotide identity shared between PVY-Bzh-1 and PVY Guiding. The development of new variants through mutation and recombination had foster global distribution of the isolates [33]. The phylogenetic analysis of the TMV isolates also confirms the redistribution of the plant viruses across borders. TMV-Xch-2 (KX650854) form cluster with the Pertunia isolate (AB369275) from South Korea while TMV-Xch-Wh (KX650855) is clustered with Shanxi (JF920727) also from China. However, TMV-Dzh-Qyg (KX650856) is rooted independently in the tree (Fig. 4) forming a branch leading to the clade but closest to the Pertunia and Shanxi isolates as well, sharing $85 \%$ nucleotide identity respectively.

The Chilli venial mottle virus isolate CVMV-Dzh-Qyg (KX650863) and PeMV-Xch (KX650857) identified in this study has $93 \%$ and $94.18 \%$ nucleotide identity with isolate Yp8 and PepMoVgp1 (NC_001517) respectively. The partial sequence of a Brassica yellow virus isolate BrYV-Xch-Wh clustered with the Brassica yellow virus (NC_016038.1) belonging to the genus Polerovirus. The analysis suggest that reassortment may have been a major event in the evolution of some of the strains and are derived from common recent ancestors that had passed through a bottleneck event. Phylogenetic analysis showed that, among the 22 isolates described worldwide, 14 Chinese isolates clustered together into an independent clade based on the near-complete genome nucleotide.

\section{Recombination events}

The major source of variability in RNA viruses are mutation, re-assortment and recombination [34] which can result in insertion of unrelated sequence elements, as well as exchange, duplication or deletion of existing viral sequence elements. Segmental recombination events of the isolates were analyzed based on at least four positive statistical methods implemented in the RDP4 program used for the analysis. Recombination breakpoints detected by the methods were recognized in the cucumovirus and potyvirus strains (Additional file 1: Figure S7a 


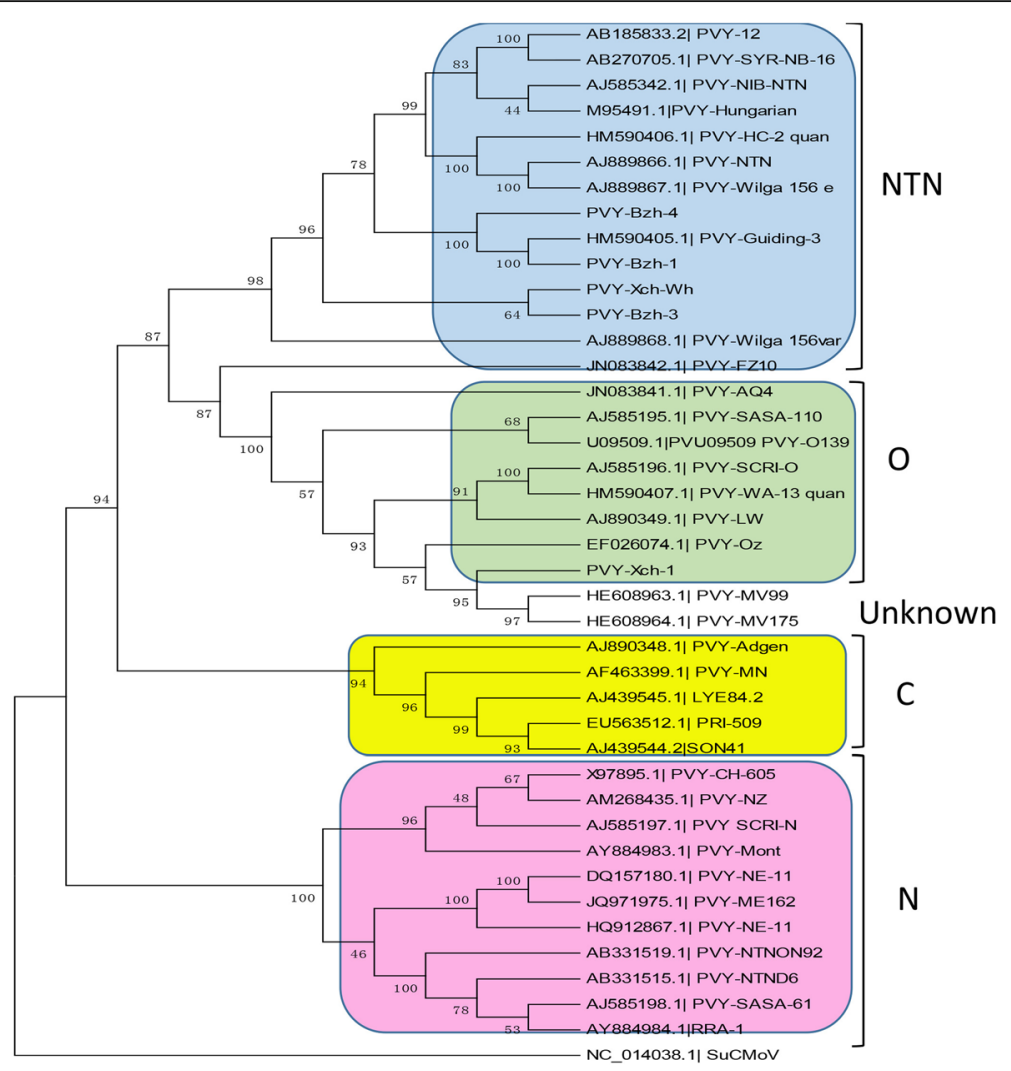

Fig. 3 Phylogenetic analyses of Potato virus $Y$ isolates and selected strains, with aligned nucleotide sequences, generated using the neighbor-joining method and MEGA6 software. The percentage of replicate trees in which the associated taxa clustered together in the bootstrap test (1000 replicates) is shown next to the branches. The tree of the Potyvirus was rooted using Sunflower chlorotic mottle virus (SuCMoV) as an out group sequence for the phylogenetic analysis

and $\mathrm{b})$. Some of the new isolates were predicted to have experienced recombination events supported by at least four programs. The major parent of PVY-Xch-Wh (KX650859) is SCRI-O isolate from United Kingdom while the minor parent is LYE84.2 from Spain. Recombination analysis in other isolates such as the PVY-Bzh-3 (KX650861) detected that the major and minor parent is WA-13 quan (China) and NE-11 (USA) isolate respectively while the PVY-Bzh-3 isolate was determined to be a recombinant of PVY-Bzh-4 and PVY-Xch-Wh. Fewer break points were observed in the cucumber virus isolates. The LS strain and FNY (USA) are the major and minor parent for CMV-Bzh-2 (KX650850) while other isolates share their origin from the Ly strain from Australia (Additional file 1: Figure S7a).

\section{Discussion}

In spite of the different plant disease control mechanisms, plant virus still cause significant economic losses in tobacco production every year in China [14]. The effectiveness of disease control strategies can be affected by the genetic exchange and changes in composition of virus population [35]. Therefore, prompt identification of invading plant virus, elucidation of the molecular determinants and genetic diversities involved in pathogenesis is important to better understand plant-pathogen isolates. In this study, our goal was to survey tobacco plant in Anhui province of China for virus infection that caused devastating infection in the fields and also capture the genetic diversity and molecular variability of the different isolates identified across the province as well as determine the effectiveness of the application of next generation sequencing technology coupled with molecular techniques in discovery of plant viruses, without the prior knowledge of the virus.

We describe a bioinformatics pipeline to efficiently identify viruses in a mixed infection of tobacco and to differentiate different strains infecting the plant across the province. The bioinformatics method is based on the deep sequencing and de novo assembly of siRNAs. The assembled contigs generated from nine sRNA libraries were analyzed for the identification of the viruses associated with Tobacco. We determined the genome sequences of 22 isolates of plant viruses infecting tobacco collected from various regions across Anhui Province of China and validated through RT-PCR and Sanger sequencing. These 


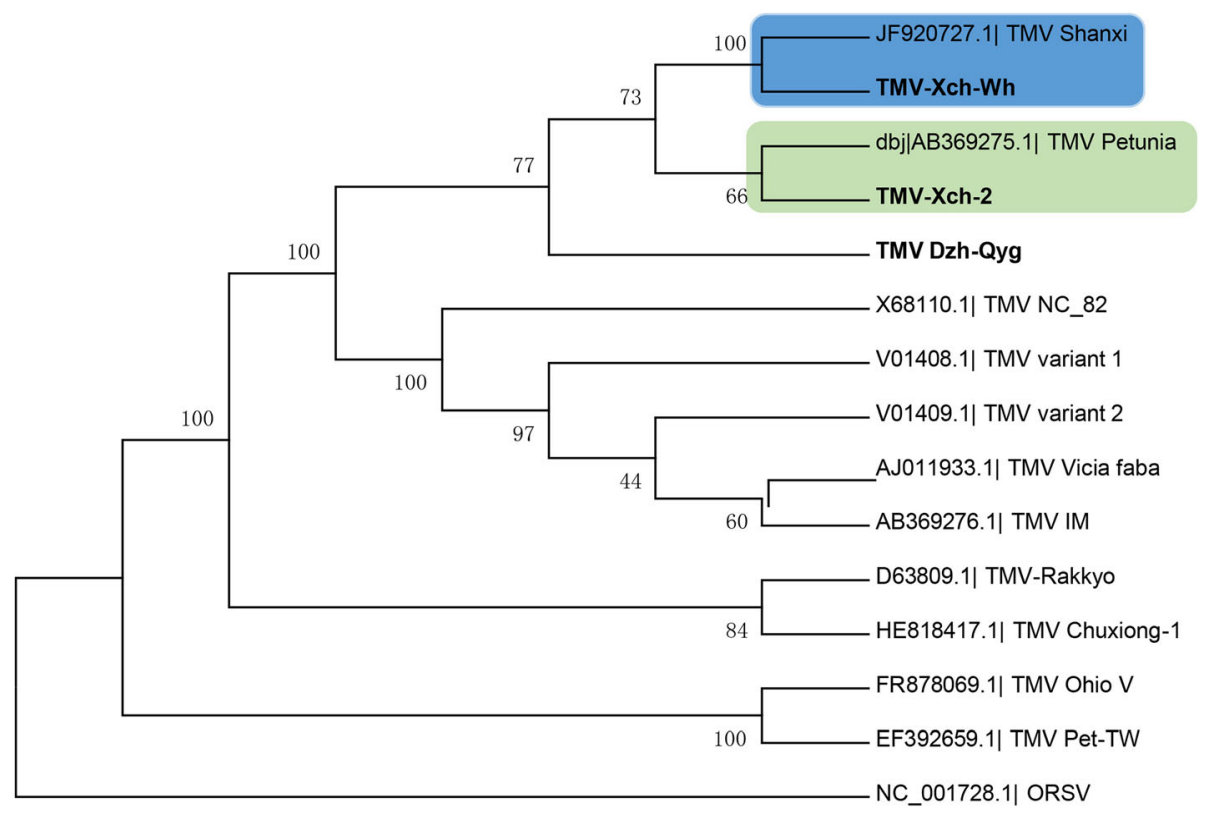

Fig. 4 Phylogenetic analyses of Tobacco mosaic virus isolates and selected strains, with aligned nucleotide sequences, generated using the neighbor-joining method and MEGA6 software. The percentage of replicate trees in which the associated taxa clustered together in the bootstrap test (1000 replicates) is shown next to the branches. The tree was rooted using Odontoglossum ring spot virus (ORSV) as an out group sequence for the phylogenetic analysis

identified viruses consist of 7 isolates of Cucumber Mosaic Virus, 5 isolates of Potato Virus Y, 3 isolates of Tobacco Mosaic Virus, 3 isolates of Tobacco Vein Banding Mosaic Virus, 1 isolate each of Pepper Mottle Virus, Brassica Yellow Virus, Chilli venial mottle virus, Broad Bean Wilt Virus 2 infecting Tobacco, a crop plant of paramount economic value. There are more isolates of CMV and PVY infecting Tobacco, compared to other identified isolates. This can be attributed to the diversity of the isolates [36] which has been reported in other parts of the world to be a serious concern to crop production.

Sequencing of the libraries of small RNA isolated from infected leaves shed more light on the consortium of replicating virus in the plant sample and proved decisive for the identification of the novel isolates [7, 9]. De novo assembly of the siRNA and BLAST search of assembled contigs to the non-redundant nucleotide and protein database identified virus sequence with more than $90 \%$ similarity. To elucidate the molecular and genetic diversity of the isolates in Anhui province of China, we analyzed the isolates and sequences of previously reported recombinant and non-recombinant isolates [36-38] retrieved from the GenBank. Some of the isolates had experienced various recombination events which are similar to other strains from other parts of the world. They were clustered in the same subgroup with other strains of viruses prevalent in other parts of the world [36, 38, 39]. The CMV isolates identified in this study showed that subgroup I is of greater prevalence than subgroup II in
China. The detected several subgroup IB isolates among historic CMV isolates and phylogenetic analysis further revealed presence of this specific subgroup in other parts of the world [31]. PVY is also considered as one of the most dangerous plant virus with different strains causing about $80 \%$ of plant losses [23] which are dependent on infecting strains, time of infection and co-infecting species. The recombination events in plants plays a critical role in the virulence of plant viruses by generating genetic variation and producing new viruses [40]. The designation of PVY strain groups is based on the biological differences of the PVY strains to overcome resistance genes in tobacco and also allow the invasion of other plant viruses by suppressing the immune response of the plant at different strain groups.

Viral evolution and host adaptation are best understood by examining the role of recombination in generating and eliminating variation in viral sequences. RNA viral replicates, apparently lack proof-reading ability and as a consequence, the frequency of mutations is much higher than in organisms with a DNA genome [41]. The recombination events in some of the isolates are as a result of mutation and genetic reassortment which has been previously reported in other isolates [36, 42, 43].

The coverage, dispersal and complexity of virus population detected in this study, calls for a need for a constant survey of not only symptomatic crops but also other crops used in mixed cropping, and proper monitoring of disease spread and efficient management. Also a fast and efficient detection method, as the Next generation sequencing that 
do not need a prior knowledge of the virus should be employed to identify viruses. Deep sequencing, bioinformatics and phylogenetic analysis, as well as comparison of the different virus species identified in Tobacco presents an important revelation of molecular variability of viruses causing devastating effects on the crop. Furthermore the proliferation of new genetic types signals a high risk for crops that must be addressed with efficient viral control and diagnostic methods.

\section{Conclusion}

In this study we describe the discovery of a consortium of plant viruses infecting Tobacco that are broadly distributed in Anhui province of China. We further characterized the genome of the 22 isolates, its variability and the siRNAs induced in tobacco plant in response to virus infection. Our result showed the effectiveness of the custom made bioinformatics pipeline coupled with molecular techniques and phylogenetic analysis, in diagnostics and identification of plant virus. Survey of plant viruses and prompt diagnostics should be frequently carried out in areas known for large cultivation of economically important crops.

\section{Methods}

\section{Collection and preparation of samples}

A field survey of the potential viral pathogens of Tobacco was conducted across farm fields in Anhui Province of China (Fig. 5a). One hundred and four symptomatic (mosaic, mottling, yellowing, ring spots, stunting, Shoestring and deformation)(Fig. 5b) leaf samples of cultivated Tobacco (Nicotina tobaccum) were collected from different regions (Xuancheng, Wuhu, Dongzhi, Qingyang, Bozhou) of Anhui province. (Tables 1). Leaf samples were immediately frozen in liquid nitrogen and stored at $-80{ }^{\circ} \mathrm{C}$ until RNA extraction.

\section{Preparation of total RNA, small RNA library construction and sequencing}

Total RNA was extracted from leaf samples as earlier described [16]. Briefly, the frozen leaves of symptomatic samples were pulverized in liquid nitrogen and suspended in $1.5 \mathrm{ml}$ tube containing extraction buffer (50 mM Tris-HCl, pH 8.0; $150 \mathrm{mM} \mathrm{LiCl;} 5 \mathrm{mM}$ EDTA, pH 8.0; 5 \% SDS, 5 \% PVP). The supernatant was treated with $2.5 \mathrm{M}$ Potassium Acetate $(\mathrm{pH} 4.8)$ followed by Chloroform : isoamyl alcohol $(24: 1 \mathrm{v} / \mathrm{v})$. The pellets were washed in $70 \%$ ethanol, air dried and re-suspended in nuclease-free water. Integrity of the RNA was verified in ethidium-bromide stained $1.2 \%$ agarose gels after electrophoresis, and purity was assessed by measuring the absorbance ratio (1.8-2.0) at $\mathrm{A}_{260} / \mathrm{A}_{280} \mathrm{~nm}$ using a Eppendorf BioPhotometer Plus (Germany). Total RNA from symptomatic leaf samples were pooled for small RNA library construction based on the site of collection as previously described [44] and deep sequencing was performed on an Illumina HiSeq-2000 sequencing platform (BGI-ShenZhen, China) using the manufacturer's instruction for sequencing.

\section{Bioinformatics analysis}

The sRNA sequence processing, assembly, and virus genome identification were conducted using a custom bioinformatics pipeline (Fig. 2). Briefly, quality of the raw Illumina sRNA reads were screened using the fastqc (http://www.bioinformatics.babraham.ac.uk/projects/fastqc) and the adapter sequence trimmed with the Cutadapt software (version 1.3) (https://cutadapt.readthedocs.io/ en/stable/). Low quality reads were cleaned with an inhouse Perl script. Trimmed sRNA sequences shorter than $18 \mathrm{nt}$ and longer than $30 \mathrm{nt}$ were discarded. The clean sRNAs reads from each sample were assembled de novo into contigs based on De Bruijin graphs with a $k$-mer length of 17 using Velvet [18] and Oases program downloaded from the European Bioinformatics Institute (http://www.ebi.ac.uk/ zerbino/oases/). The assembled contigs were used to query the non-redundant GenBank nucleotide and protein databases, respectively, using the BLAST program [45]. Highest hits having $\geq 90 \%$ coverage and identity with the nucleotide sequences in the non-redundant NCBI database were extracted for further analysis using BLASTn (e-value cutoff: $10^{-3}$ ). Furthermore, they were filtered and searched against the non-redundant protein database of NCBI by BLASTx and tblastx to compare the homology, as short sequences may lead to false positive results [46]. Final hits with nucleic acid and protein coverage $\geq 90 \%$ and identity $\geq 90 \%$ transcription of the virus were identified and aligned to references in order to determine their orientation and positions. The small RNAs of the hits were mapped to the to the closest distantly related viral genome with Bowtie [20] to determine the distribution and abundance of the sRNA.

\section{RT-PCR, cloning, full-length genome amplification, and sequencing}

Reverse transcription PCR (RT-PCR) was performed using primers (Table S1) designed from sequences of specific assembled contigs to bridge the gaps between them, so as to obtain the near-complete genome sequences. The PCR product segments were electrophoresed in $1.0 \%$ agarose gels, bands were excised and purified using the SanPrep Gel Extraction Kit (Sangon Biotech, China). The amplification products were ligated to pMD18-T vector (Takara Biotechnology Co., Ltd), transformed into Escherichia coli $\mathrm{DH} 5 \alpha$ and positive clones were sequenced. Two clones from independent PCR reactions 


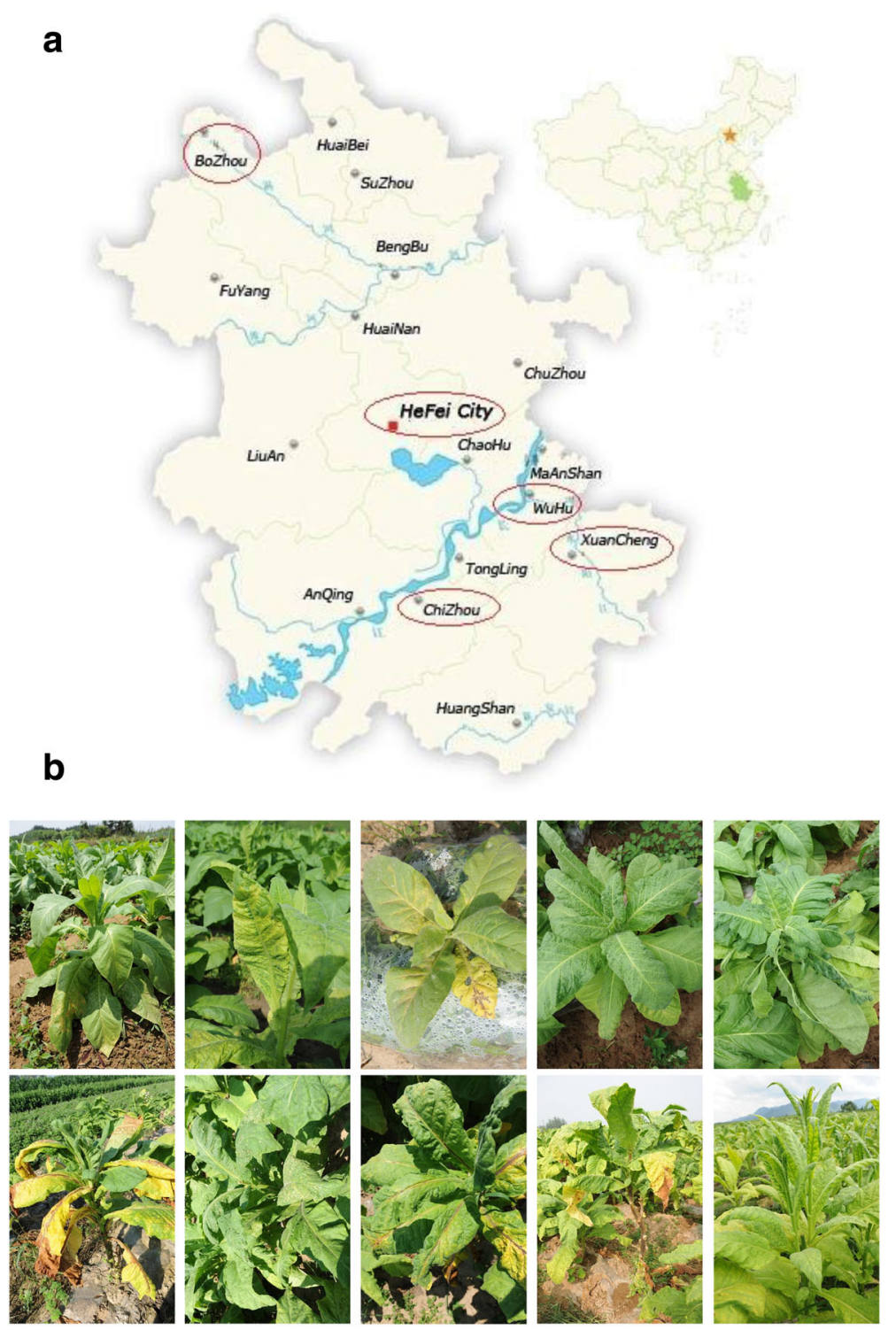

Fig. 5 a Sampling areas of infected tobacco plants in Anhui Province of China. b Typical view representation of yellow mosaic mosaic, stunting, Shoestring and deformation symptom of the overall Tobacco leaf samples collected across Anhui Province

products were sequenced twice on both strands for each amplified fragment through the conventional Sanger dideoxy sequencing.

\section{Phylogenetic analysis}

ORFs were predicted using ORF Finder. Conserved domains/motifs were analyzed using SMART [12]. The new sequences were aligned with other sequences retrieved from the GenBank with Mafft [47] and phylogenetic analyses were conducted using the neighbor-joining method by MEGA6 [48], with the bootstrap of 1000 replicates [49]. Maximum likelihood resulted in similar phylogenetic trees. Other isolates were retrieved from the GenBank database for the phylogenetic analysis. Origin and accession number of the isolates are listed in the Additional file 2: Table S2, S3 and S4.

\section{Recombination analysis}

The putative recombination events among the isolates and genus member were evaluated using the Recombination Detection Program v.4.66 (RDP4) [50] made up of different methods namely recombination detection program (RDP), statistical tests for detecting gene conversion (GENECONV) [51], BOOTSCAN [52], MaxiChi [53], Chimera [54], SiScan [55], and 3SEQ [56], implemented in the RDP Beta 4.66 program. Alignments of nucleotide sequences produced in Mafft [47] and MEGA6 [48] were used in RDP4 with the default settings $(0.05 p$ - 
value cut-off). Recombination events were considered as significant if more than four algorithms had a consensus $P$-value $\leq 0.01$, in addition to phylogenetic evidence of recombination. Otherwise, they were regarded as 'tentative' recombination events [57].

\section{Additional files}

Additional file 1: Figure S1. 21 and 24 nt peak distribution of the libraries. Figure $\mathbf{S} \mathbf{2}(\mathbf{a}, \mathbf{b}, \mathbf{c})$. Length distribution of total sRNAs (18 to $30 \mathrm{nt}$ ) in the nine libraries. Figure S3. Distribution of siRNA Reads on Reference Genome shows coverage across the reference. Figure S4. Phylogenetic analyses of isolates of the genus Potyvirus and some selected group members based on complete genome sequences, generated using the neighbor-joining method and MEGA6 software. The percentage of replicate trees in which the associated taxa clustered together in the bootstrap test (1000 replicates) is shown next to the branches. Figure S5. Phylogenetic analyses of CMV Isolates and selected cucumoviruses based on complete genome sequences, generated using the neighbor-joining method and MEGA6 software. The percentage of replicate trees in which the associated taxa clustered together in the bootstrap test (1000 replicates) is shown next to the branches. Figure S6. Phylogenetic analyses of isolates of the genus Poleroviruss (A) and Tobamovirus (B) based on complete genome sequences, generated using the neighbor-joining method and MEGA6 software. The percentage of replicate trees in which the associated taxa clustered together in the bootstrap test (1000 replicates) is shown next to the branches. Figure S7a and $\mathbf{b}$. Putative recombination event involving (a) Potato virus $Y$ and (b) Cucumber mosaic virus isolates from Tobacco calculated by Recombination Detection Program v. 4.16. (PPTX 466 kb)

Additional file 2: Table S1. Primer sets used in this study for RT-PCR to bridge the gaps between the assembled contigs of each isolates. Table S2. Strain, accession numbers and origin of reported Cucumber mosaic virus isolates used for phylogenetic comparison of sequence in this study. Table S3. Strain, accession numbers and origin of reported Potato virus $Y$ isolates used for phylogenetic comparison of sequence in this study. Table S4. Strain, accession numbers and origin of reported TMV isolates retrieved from Genbank used for phylogenetic comparison and recombination analysis of sequence in this study. (DOCX $25 \mathrm{~kb}$ )

\section{Abbreviations}

Ago: Argonaut protein; BBWV: Broad bean wilt virus 2; BrYV: Brassica yellow virus; CMV: Cucumber mosaic virus; CVMV: Chilli venial mottle virus; DCL: Dicer-like ribonuclease; dsRNA: double stranded RNA; ELISA: Enzyme-linked immunosorbent assay; NGS: Next Generation Sequencing; PCR: Polymerase chain reaction; PeMV: Pepper mottle virus; PV: Potato virus Y; RISC: RNA-induced silencing complex; siRNAs: small-interfering RNAs; TMV: Tobacco mosaic virus; TVBMV: Tobacco vein banding Mosaic virus; vsRNAs: viral small RNAs

\section{Acknowledgements}

This study was supported by grants from the Chinese National Natural Science Foundation (no. 31272011), Key Program of Anhui Province Tobacco Monopoly Administration (no. 20150551007) and Natural Science Foundation of Anhui Province NO.1608085QC59. We will like to thank the support of Chinese Academy of Science - The World Academy of Science (CAS-TWAS) PhD President's Fellowship to Ibukun A. Akinyemi. The funders played no role in the study design, data collection and analysis, decision to publish, or preparation of the manuscript.

\section{Availability of data and materials}

The genome sequence of the 22 virus isolates identified in this study have been deposited in GenBank and allocated the accession numbers of KX650847-KX650868.

\section{Authors' contributions}

QW conceived the study and designed the project. BZ and FW collected the samples. IAA, FW, BZ and SQ conducted the experiments. IAA carried out the bioinformatics analysis. IAA and QW analyzed the data, wrote and revised the manuscript. All authors read and approved the final manuscript.

\section{Competing interests}

The authors declare that they have no competing interests.

\section{Consent for publication}

Not applicable.

Ethics approval and consent to participate

Not applicable.

\section{Author details}

${ }^{1}$ School of Life Sciences, University of Science and Technology of China, Hefei, Anhui 230027, China. ${ }^{2}$ Tobacco Research Institute, Anhui Academy of Agricultural Sciences, Hefei, Anhui 230031, China.

Received: 4 August 2016 Accepted: 18 October 2016 Published online: 04 November 2016

\section{References}

1. Nicaise V. Crop immunity against viruses: outcomes and future challenges. Front Plant Sci. 2014;5:660.

2. Adriaenssens EM, Cowan DA. Using signature genes as tools to assess environmental viral ecology and diversity. Appl Environ Microbiol. 2014:80:4470-80.

3. Domingo E, Sheldon J, Perales C. Viral quasispecies evolution. Microbiol Mol Biol Rev. 2012;76:159-216.

4. Brodersen $P$, Voinnet $O$. The diversity of RNA silencing pathways in plants. Trends Genet. 2006;22:268-80.

5. Covey SN, AlKaff NS, Langara A, Turner DS. Plants combat infection by gene silencing. Nature. 1997;385:781-2.

6. Roy A, Shao J. A case study on discovery of novel citrus leprosis virus cytoplasmic type 2 utilizing small RNA libraries by next generation sequencing and bioinformatic analyses. Journal of Data Mining in Genomics \& Proteomics. 2013;04.

7. Wu Q, Ding SW, Zhang Y, Zhu S. Identification of viruses and viroids by next-generation sequencing and homology-dependent and homologyindependent algorithms. Annu Rev Phytopathol. 2015;53:425-44.

8. Al Rwahnih M, Dave A, Anderson MM, Rowhani A, Uyemoto JK, Sudarshana MR. Association of a DNA virus with grapevines affected by red blotch disease in California. Phytopathology. 2013;103:1069-76.

9. Giampetruzzi A, Roumi V, Roberto R, Malossini U, Yoshikawa N, La Notte P Terlizzi F, Credi R, Saldarelli P. A new grapevine virus discovered by deep sequencing of virus- and viroid-derived small RNAs in Cv Pinot gris. Virus Res. 2012:163:262-8.

10. Maliogka VI, Olmos A, Pappi PG, Lotos L, Efthimiou K, Grammatikaki G, Candresse T, Katis NI, Avgelis AD. A novel grapevine badnavirus is associated with the Roditis leaf discoloration disease. Virus Res. 2015;203:47-55.

11. Kreuze JF, Perez A, Untiveros M, Quispe D, Fuentes S, Barker I, Simon R. Complete viral genome sequence and discovery of novel viruses by deep sequencing of small RNAs. A generic method for diagnosis, discovery and sequencing of viruses. Virology. 2009;388:1-7.

12. Warner KE. The economics of tobacco: myths and realities. Tob Control. 2000;9:78-89.

13. Ding M, Yang C, Zhang L, Jiang ZL, Fang Q, Qin XY, Zhang ZK. Occurrence of Chilli veinal mottle virus in Nicotiana tabacum in Yunnan. China Plant Disease. 2011;95:357.

14. Tian YP, Liu JL, Zhang CL, Liu YY, Wang B, Li XD, Guo ZK, Valkonen JP. Genetic diversity of potato virus $Y$ infecting tobacco crops in China. Phytopathology. 2011;101:377-87.

15. Dong JH, Yin YY, Xu XY, Duan YM, Zhang ZK. First report of tomato spotted wilt virus in tomato and tobacco in China. J Plant Pathol. 2010;92:S121.

16. Wang F, Qi S, Gao Z, Akinyemi IA, Xu D, Zhou B. Complete genome sequence of tobacco virus 1, a closterovirus from Nicotiana tabacum. Arch Virol. 2016;161:1087-90.

17. Q-F LIU, M-D LI, H-Y WU, X-D WU, D-Y PENG. Geochemical characteristics of typical tobacco-planting soils in Zhangjiajie mountainous area. Chin J Eco-Agric. 2012;6:19

18. Zerbino DR, Birney E. Velvet: algorithms for de novo short read assembly using de Bruijn graphs. Genome Res. 2008;18:821-9. 
19. Schulz MH, Zerbino DR, Vingron M, Birney E. Oases: robust de novo RNAseq assembly across the dynamic range of expression levels. Bioinformatics. 2012;28:1086-92.

20. Langmead B, Trapnell C, Pop M, Salzberg SL. Ultrafast and memory-efficient alignment of short DNA sequences to the human genome. Genome Biol. 2009;10:R25.

21. Kayode AB, Odu BO, Ako-Nai KA, Alabi OJ. Occurrence of cucumber mosaic virus subgroups IA and IB isolates in tomatoes in Nigeria. Plant Dis. 2014;98:1750

22. Chen YF, Chen JS, Zhang HR, Tang XS, Du ZY. Molecular evidence and sequence analysis of a natural reassortant between Cucumber mosaic virus subgroup IA and II strains. Virus Genes. 2007;35:405-13.

23. Pourrahim R, Farzadfar S. Population analysis of Iranian potato virus $Y$ isolates using complete genome sequence. Plant Pathol J. 2016;32:33-46.

24. Vance VB, Moore D, Turpen TH, Bracker A, Hollowell VC. The complete nucleotide sequence of pepper mottle virus genomic RNA: comparison of the encoded polyprotein with those of other sequenced potyviruses. Virology. 1992;191:19-30.

25. Adams MJ, Antoniw JF, Kreuze J. Virgaviridae: a new family of rod-shaped plant viruses. Arch Virol. 2009;154:1967-72.

26. Bonnet J, Fraile A, Sacristan S, Malpica JM, Garcia-Arenal F. Role of recombination in the evolution of natural populations of Cucumber mosaic virus, a tripartite RNA plant virus. Virology. 2005:332:359-68.

27. Davino S, Panno S, Rangel EA, Davino M, Bellardi MG, Rubio L. Population genetics of cucumber mosaic virus infecting medicinal, aromatic and ornamental plants from northern Italy. Arch Virol. 2012;157:739-45.

28. Moury B, Morel C, Johansen E, Jacquemond M. Evidence for diversifying selection in Potato virus $Y$ and in the coat protein of other potyviruses. Gen Virol. 2002;83:2563-73.

29. Singh RP, Valkonen JP, Gray SM, Boonham N, Jones RA, Kerlan C, Schubert J. Discussion paper: the naming of potato virus $Y$ strains infecting potato. Arch Virol. 2008;153:1-13.

30. Glais L, Tribodet M, Kerlan C. Genomic variability in Potato potyvirus Y (PVY): evidence that PVY(N)W and PVY(NTN) variants are single to multiple recombinants between $\mathrm{PVY}(\mathrm{O})$ and $\mathrm{PVY}(\mathrm{N})$ isolates. Arch Virol. 2002:147:363-78

31. Cuevas JM, Delaunay A, Visser JC, Bellstedt DU, Jacquot E, Elena SF. Phylogeography and molecular evolution of potato virus Y. PLoS One. 2012;7:e37853.

32. Domingo E, Escarmis C, Sevilla N, Moya A, Elena SF, Quer J, Novella IS, Holland J. Basic concepts in RNA virus evolution. Faseb Journal. 1996;10:859-64.

33. Hu XJ, Karasev AV, Brown CJ, Lorenzen JH. Sequence characteristics of potato virus Y recombinants. J Gen Virol. 2009;90:3033-41.

34. Worobey $\mathrm{M}$, Holmes $\mathrm{EC}$. Evolutionary aspects of recombination in RNA viruses. J Gen Virol. 1999;80:2535-43.

35. Acosta-Leal R, Duffy S, Xiong Z, Hammond RW, Elena SF. Advances in plant virus evolution: translating evolutionary insights into better disease management. Phytopathology. 2011;101:1136-48.

36. Moodley V, Ibaba JD, Naidoo R, Gubba A. Full-genome analyses of a Potato Virus Y (PVY) isolate infecting pepper (Capsicum annuum L.) in the Republic of South Africa. Virus Genes. 2014;49:466-76.

37. Wang F, Gao ZL, An MN, Zhou BG, Wu YH. Sequencing and phylogenetic analysis of potato virus $Y$ Liaoning isolate in China. J Integr Agric. 2013;12:1195-200.

38. Nouri S, Arevalo R, Falk BW, Groves RL. Genetic structure and molecular variability of Cucumber mosaic virus isolates in the United States. PLoS One. 2014;9, e96582.

39. Kehoe MA, Coutts BA, Buirchell BJ, Jones RAC. Plant virology and next generation sequencing: experiences with a Potyvirus. Plos One. 2014;9.

40. Froissart R, Roze D, Uzest M, Galibert L, Blanc S, Michalakis Y. Recombination every day: abundant recombination in a virus during a single multi-cellular host infection. PLoS Biol. 2005;3:e89.

41. Malpica JM, Fraile A, Moreno I, Obies Cl, Drake JW, Garcia-Arenal F. The rate and character of spontaneous mutation in an RNA virus. Genetics. 2002;162:1505-11.

42. Roossinck MJ. Evolutionary history of Cucumber mosaic virus deduced by phylogenetic analyses. J Virol. 2002;76:3382-7.

43. Lin HX, Rubio L, Smythe AB, Falk BW. Molecular population genetics of Cucumber mosaic virus in California: evidence for founder effects and reassortment. J Virol. 2004:78:6666-75.
44. Yang X, Wang Y, Guo W, Xie Y, Xie Q, Fan L, Zhou X. Characterization of small interfering RNAs derived from the geminivirus/betasatellite complex using deep sequencing. PLoS One. 2011;6, e16928.

45. Altschul SF, Gish W, Miller W, Myers EW, Lipman DJ. Basic local alignment search tool. J Mol Biol. 1990;215:403-10.

46. Ho T, Tzanetakis IE. Development of a virus detection and discovery pipeline using next generation sequencing. Virology. 2014;471:54-60.

47. Katoh K, Standley DM. MAFFT multiple sequence alignment software version 7: improvements in performance and usability. Mol Biol Evol. 2013;30:772-80.

48. Tamura K, Stecher G, Peterson D, Filipski A, Kumar S. MEGA6: molecular evolutionary genetics analysis version 6.0. Mol Biol Evol. 2013;30:2725-9.

49. Felsenstein J. Confidence-limits on phylogenies - an approach using the bootstrap. Evolution. 1985;39:783-91.

50. Martin DP, Murrell B, Golden M, Khoosal A, Muhire B. RDP4: Detection and analysis of recombination patterns in virus genomes. Virus Evolution. 2015;1:vev003.

51. Padidam M, Sawyer S, Fauquet CM. Possible emergence of new geminiviruses by frequent recombination. Virology. 1999;265:218-25.

52. Salminen MO, Carr JK, Burke DS, McCutchan FE. Identification of breakpoints in intergenotypic recombinants of HIV type 1 by bootscanning. AIDS Res Hum Retroviruses. 1995;11:1423-5.

53. Smith JM. Analyzing the mosaic structure of genes. J Mol Evol. 1992;34:126-9.

54. Posada D, Crandall KA. Evaluation of methods for detecting recombination from DNA sequences: computer simulations. Proc Natl Acad Sci U S A. 2001;98:13757-62.

55. Gibbs MJ, Armstrong JS, Gibbs AJ. Sister-Scanning: a Monte Carlo procedure for assessing signals in recombinant sequences. Bioinformatics. 2000;16:573-82.

56. Boni MF, Posada D, Feldman MW. An exact nonparametric method for inferring mosaic structure in sequence triplets. Genetics. 2007;176:1035-47.

57. Tomitaka Y, Ohshima K. A phylogeographical study of the Turnip mosaic virus population in East Asia reveals an 'emergent" lineage in Japan. Mol Ecol. 2006;15:4437-57.

\section{Submit your next manuscript to BioMed Central and we will help you at every step:}

- We accept pre-submission inquiries

- Our selector tool helps you to find the most relevant journal

- We provide round the clock customer support

- Convenient online submission

- Thorough peer review

- Inclusion in PubMed and all major indexing services

- Maximum visibility for your research

Submit your manuscript at www.biomedcentral.com/submit 